Folia primat. 1971;14(Suppl 1):103

\title{
VII. Summary
}

The embryonal development of the hand-skeleton of Tupaia glis and Microcebus murinus was studied. Skeleton material from adult animals of the same kind and also of selected insectivora, rodentia, and primates was consulted to supplement the study.

On the basis of the differences determined between the hand-skeletons of Tupaia and

Microcebus, two types of hands are defined: the spread hand and the prehensile hand. The spread hand, such as to some extent materialised in Tupaia, is distinguished by the bowl-shaped formation of the elements of its skeleton.

The prehensile hand, as formed in Microcebus, is distinguished by an orientation and formation of the rows of wrist-bones towards proximo-distal.

Because of its skeletal-morphologic properties, the spread hand permits the variable abduction of the wrist, the metacarpus, and the fingers. It permits quadrupedal locomotion in the groundorientated milieus of the bush-and tree-zone that can be counted as semi-arboricol.

The prehensile hand, because of its skeletal-morphologic properties, guarantees at least a pseudoopposition of the thumb and thus provides the basic prerequisite for a true arboricolic life. 